\title{
EFFECT OF SOLUBLISING AIDS ON THE ENTRAPMENT OF LORATIDINE IN PRE-FABRICATED PVA FILAMENTS USED FOR FDM BASED 3D-PRINTING
}

\author{
FAISAL MAHMOOD ${ }^{1}$, AMJAD HUSSAIN ${ }^{1 *}$, MUHAMMAD SOHAIL ARSHAD ${ }^{2}$, NASIR ABBAS ${ }^{1}$, \\ MUHAMMAD IRFAN ${ }^{3}$, NADIA QAMAR ${ }^{1}$, FAHAD HUSSAIN $^{1}$ \\ and MUHAMMAD USMAN GHORI ${ }^{4}$
}

${ }^{1}$ University College of Pharmacy, University of the Punjab, Lahore, Pakistan ${ }^{2}$ Faculty of Pharmacy, Bahauddin Zakariya University, Multan, Pakistan ${ }^{3}$ Department of Pharmaceutics, Faculty of Pharmaceutical Sciences, GC University, Faisalabad, Pakistan ${ }^{4}$ Department of Pharmacy, School of applied Sciences, University of Huddersfield, Huddersfield, United Kingdom

\begin{abstract}
Low drug loading efficiency is the limiting factor in the use of pre-fabricated filaments for 3D printing of pharmaceuticals. The aim of the present study was to modify the material properties of pre-fabricated filament by incorporating suitable solubilizing aids in order to enhance the drug loading efficiency. Loratadine was loaded into PVA filaments by using solubilizers (Soluplus ${ }^{\circledR}$, Sodium lauryl sulfate) and plasticizers (glycerin and Polyethylene glycol-400) and the printability of filaments was investigated. The treated filaments were characterized for morphology and diameter changes, drug content, FTIR and thermal properties and printed into tablets of suitable dimensions. The printed tablets were also characterized for drug assay and drug release. The results have shown that the surface of different drug-loaded filaments becomes rough with almost no change in diameter hence, these filaments remained printable. However, there was 7 to 24 times enhancement in drug content of treated filaments with particularly those pretreated with glycerin and soaked in drug solution containing Soluplus. The printed tablets have also shown almost similar drug content as their precursor filaments and the release followed diffusion mechanism in most of the formulations. The study concludes that the treatment of PVA filament with solubilizer aids has significantly improved drug loading without compromising the printability.
\end{abstract}

Keywords: Fused deposition modeling (FDM), 3D printing, PVA filaments, plasticizers, solubilizers

Fused deposition modeling (FDM) based threedimensional printing (3DP) is amongst a recently used methods intended for the preparation of customized medicine (1). Dose customization involves; tailoring the medical treatment based on the individual characteristics, needs and preferences of each patient (2). This approach can improve the safety profile of drugs by lowering the risk of overdosing (particularly for drugs prescribed at lower doses), hence adverse reactions (3). FDM is an additive manufacturing technique (4) which constructs a 3D object directly from computer-aided design (CAD) data, utilizing a thermoplastic material (such as PVA and PLA, etc.) in the form of filament extruded through printing head of 3D printer in a layer by layer fashion (5).
The classical approach for drug loading in commercially available PVA or PLA filaments is the impregnation or solvent diffusion (6). The method involves soaking the filament in the saturated alcoholic solution of a drug at room temperature (7). The preparations reported so far have shown a meager drug loading i.e. $(<2 \% \mathrm{w} / \mathrm{w})$ by this method. In one such study, fluorescein sodium was loaded in PVA filament. Although the printed tablets were mechanically strong with low friability, they exhibited a drug content of $<1 \%$ (6). Similarly, 3D printing of 4-aminosalicylic acid (4-ASA) and 5-aminosalicylic acid (5-ASA) tablets also revealed almost similar extent of drug loading (8). The impregnation method has also been tried for 3D printing of pred-

* Corresponding author: e-mail: amjad_husein@hotmail.com 
nisolone tablets but no significant improvement in drug loading was achieved (7).

Any formulation strategy for the improvement in drug loading via soaking method has not been tried so for in literature. Rather, tailor-made filaments using suitable polymer blend with drug through Hot Melt Extrusion were fabricated (9-12). Although, this method ensures better drug loading efficiency for different drugs but repeated thermal shocks (during filament fabrication and then during printing) may cause degradation of the drug. Also, the resulting filaments do not have the desired mechanical strength and uniformity in diameter for a 3D printer (13). Therefore, pre-fabricated filaments are still useful because of their excellent inherent mechanical and thermal properties and adaptability in $3 \mathrm{D}$ printers.

The aim of the present study was to enhance the drug loading efficiency of pre-fabricated PVA filament. The inclusion of formulation aids such as solubilizer and/or plasticizers in the drug solution may be useful in this regard. In this study, the loading of loratadine from its ethanolic solution containing two solubilizers (Soluplus, Sodium lauryl Sulphate) and two plasticizers (glycerin and Polyethylene glycol400) in PVA filament was investigated.

\section{EXPERIMENTAL}

Loratadine was obtained as a gift sample from Synchro Pharma, Lahore, Pakistan. Commercially available pre-fabricated PVA filament (Makerbot ${ }^{\circledR}$ New York, US). Soluplus ${ }^{\circledR}$ a graft copolymer of polyethylene glycol, polyvinyl caprolactam, and polyvinyl acetate) (BASF, Ludwigshafen Germany), and Sodium Lauryl Sulphate (SLS) (Merck, Frankfurt, Germany), Glycerin and Polyethylene glycol (PEG-400) (China) were obtained from the local supplier. Ethanol analytical grade $(99.9 \%$ pure) was purchased from Merck, Germany.

\section{Loading of drug in PVA filament}

PVA filament ( $\sim 5 \mathrm{~g})$ was immersed in the saturated ethanolic solution of loratadine of various compositions as described in Table 1. F1 was the filament soaked in ethanolic drug solution. F2 and F3 were soaked in drug solution containing of soluplus and SLS respectively. The filaments of F4 and F5 in the first step were soaked in glycerin for $1 \mathrm{hr}$ at $25^{\circ} \mathrm{C}$ and then immersed in ethanolic drug solution containing soluplus and SLS, respectively. F6 and F7 were treated with PEG-400 at first step instead of glycerin with similar treatment at the second step. The soaked filaments were stirred at $50 \mathrm{rpm}$ for 48 hrs at room temperature $\left(25 \pm 1^{\circ} \mathrm{C}\right) . \mathrm{F} 8$ filament was heated in a microwave $(800 \mathrm{~W}$ for $2 \mathrm{~min}$ ) and then soaked in ethanolic solution of loratadine $\mathrm{HCl}$.

\section{Characterization of the PVA filament}

Loratadine loaded filaments and control (filament alone) were studied for their morphology (using a stereo-zoom microscope, Olympus, Japan) and thickness (using digital micrometer). The mechanical parameters including tensile strength, Young's modulus and stiffness of filaments were determined using a pre-calibrated Universal material testing machine (Lloyd Lf Plus series, Lloyd Instruments Ltd., Largo, FL, USA). Each filament ( $\sim 5 \mathrm{~cm}$ long) was affixed between the two jaws of machine and Tensile strength test was applied (Nexygen Plus 4 software). The jaws were moved at the speed of $250 \mathrm{~mm} / \mathrm{s}$ and the force and length at which filament break apart was measured. The values of tensile strength Young's modulus and stiffness were calculated by applying the equations 1, 2, and 3, respectively. Each measurement was recorded in triplicate and the average value was reported.

$$
\text { Tensile strength }(\sigma)=\frac{F}{A}
$$

where ' $\mathrm{F}$ ' is the force at which the filament breaks and ' $\mathrm{A}$ ' is the cross-sectional area of filament.

Table 1. Detail of pre-treatment and composition of drug solution for the loading of loratadine in PVA filament.

\begin{tabular}{|c|c|c|}
\hline Formulation code & Pretreatment & Composition of drug solution \\
\hline F1 & Nil & Drug solution alone \\
\hline F2 & Nil & Soluplus $5 \% \mathrm{w} / \mathrm{v}$ \\
\hline F3 & Nil & SLS $1 \% \mathrm{w} / \mathrm{v}$ \\
\hline F4 & Soaked in Glycerin & Soluplus $5 \% \mathrm{w} / \mathrm{v}$ \\
\hline F5 & Soaked in Glycerin & SLS $1 \% \mathrm{w} / \mathrm{v}$ \\
\hline F6 & Soaked in PEG-400 & Soluplus $5 \% \mathrm{w} / \mathrm{v}$ \\
\hline F7 & Soaked in PEG-400 & SLS $1 \% \mathrm{w} / \mathrm{v}$ \\
\hline F8 & Microwave 2 min & Drug solution alone \\
\hline
\end{tabular}




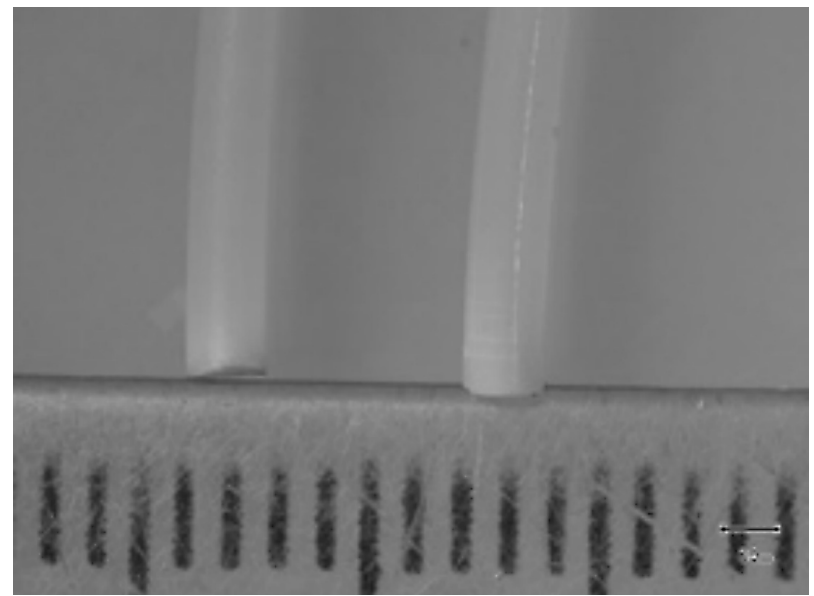

Figure 1. PVA filament (before and after soaking).

$$
\text { Young's Modulus }(E)=\frac{\sigma}{\varepsilon}
$$

where ' $\sigma$ ' is the stress applied and ' $\varepsilon$ ' is strain i.e. change in length of filament before it breaks.

$$
\operatorname{Stiffness}(k)=\frac{F}{\delta}
$$

where ' $F$ ' is the force applied and ' $\delta$ ' is displacement produced by the applied force

\section{ATR-FTIR spectroscopy}

Fourier Transform Infrared (FTIR) spectra of filaments before and after drug loading were recorded using FTIR spectrophotometer (Cary 630, Agilent Technologies, California US) equipped with a diamond ATR unit across the range of 4000-600 $\mathrm{cm}^{-1}$.

\section{Differential Scanning Calorimetry (DSC)}

DSC studies of filaments before and after drug loading were performed using a TA instrument ( $Q$ 2000, US). Powdered PVA filament ( $10 \mathrm{mg}$ ) was transferred in aluminum pan and scanned at the heating rate of $10^{\circ} \mathrm{C} \mathrm{min}{ }^{-1}$ over a temperature range of 25 to $300^{\circ} \mathrm{C}$ with under a nitrogen purge 50 $\mathrm{mL} / \mathrm{min}$. The instrument was calibrated at $10^{\circ} \mathrm{C} \mathrm{min}-$ ${ }^{1}$ using indium (fusion temperature $156.5^{\circ} \mathrm{C}$ and $\ddot{\mathrm{A}} \mathrm{H}$ $\left.28.57 \mathrm{Jg}^{-1}\right)$.

\section{Determination of drug content in the PVA fila- ments}

The drug content of each filament was determined by UV-Spectrophotometer (2550 Shimadzu, Kyoto, Japan). For this purpose, drug-loaded filament $(\sim 0.5 \mathrm{~g})$ was crushed in mortar and pestle for 5 min and then mixed in the distilled water. The mix- ture was stirred while heating at $40^{\circ} \mathrm{C}$ followed by sonication for $10 \mathrm{~min}$ and filtration. The dissolved content of loratadine was determined by measuring $\mathrm{UV}$ absorption at $254 \mathrm{~nm}\left(\delta_{\max }\right.$ of drug). The drug content per $0.5 \mathrm{~g}$ of filament was reported which was the approximate weight of tablet with given dimensions for $3 \mathrm{D}$ printing (see in the following sections). Each sample was analyzed in triplicate (n $=3$ ) and the average values were reported.

\section{Fabrication of 3D printed Tablets}

Tablets design having predefined dimensions of $13 \mathrm{~mm}$ (diameter) $\times 5 \mathrm{~mm}$ (height) were built in CAD software. These templates were printed in Replicator 2X, 3D printer (Makerbot Inc., New York, USA) using drug-loaded filaments (F1-F8) and named T1-T8, respectively. The settings of the printer were: extruder temperature $220^{\circ} \mathrm{C}$, platform temperature $115^{\circ} \mathrm{C}$, layer height $0.2 \mathrm{~mm}$, number of shells 2, extrusion speed $90 \mathrm{~mm} / \mathrm{sec}$ and traveling speed of $150 \mathrm{~mm} / \mathrm{sec}$. Tablets with infill density of $50 \%$ and hexagonal design were printed from different filaments.

\section{Characterization of the 3D Printed tablets}

Hardness and friability tests of the printed tablets were carried out according to the methods described in official books for conventional tablets (USP 2013). The hardness of 10 tablets of each type was measured using the traditional tablet hardness tester (Dawn Analyticals, Lahore Pakistan). For the measurement of friability $\sim 6.5 \mathrm{~g}$ of tablets of each formulation was weight and taken in the drum of friabilator (Western Analytical Services, Lahore, Pakistan). The drum was rotated at $25 \mathrm{rpm}$ for $4 \mathrm{~min}$ 
and tablets were reweighed at the end of the test. Drug content assay in each tablet was determined using UV spectrophotometry as described for filament.

The release of Loratadine from the 3D printed tablets was determined using USP dissolution apparatus II (DAWN Scientific, Pakistan) in phosphate buffer pH 6.8 (volume: $900 \mathrm{~mL}$, maintained at temperature: $37 \pm 0.4^{\circ} \mathrm{C}$ ) and a rotation speed of $50 \mathrm{rpm}$. The aliquots of $5 \mathrm{~mL}$ were withdrawn at time intervals of $5,15,30,45$ and $60 \mathrm{~min}$ and replaced with fresh dissolution medium in order to maintain the sink conditions. The drug concentration in samples was determined by UV -spectrophotometer (2550 Shimadzu, Japan) at $254 \mathrm{~nm}$ and percent drug release was determined using a calibration curve.

\section{RESULTS}

\section{Characteristics of PVA filaments}

The morphological appearance of PVA filament before and after drug loading was described in
Figure 1. The blank filament was white in color, relatively tough, with a smooth surface and thickness of $1.75 \pm 0.05 \mathrm{~mm}$. The different drug-loaded filaments have a shiny, rough surface and underwent little change in diameter $1.80 \pm 0.08$. These changes in filament thickness fall within the permissible limit of the 3D printer (14).

PVA filament was stiff and exhibited the tensile strength of $20.13 \pm 0.38 \mathrm{MPa}$ and Young's modulus $1931.5 \pm 193.1 \mathrm{MPa}$ and stiffness $134.8 \pm 11.8$. After impregnation in drug solution (F1), although the tensile strength remains almost the same (19.81 \pm 0.25 ), there was $\sim 10 \%$ reduction in the values of Young's modulus and $\sim 15 \%$ reduction in stiffness values (Table 2). In treated filaments where drug solution contains solubilizer (F2-F3), Young's modulus was $\sim 15 \%$ and stiffness was $\sim 25 \%$ lower than the intact filament (Table 2). Furthermore, F4-F7, which contain plasticizers, a further $10 \%$ reduction in the values of both these parameters indicated the plasticizing property of added substances, particu-
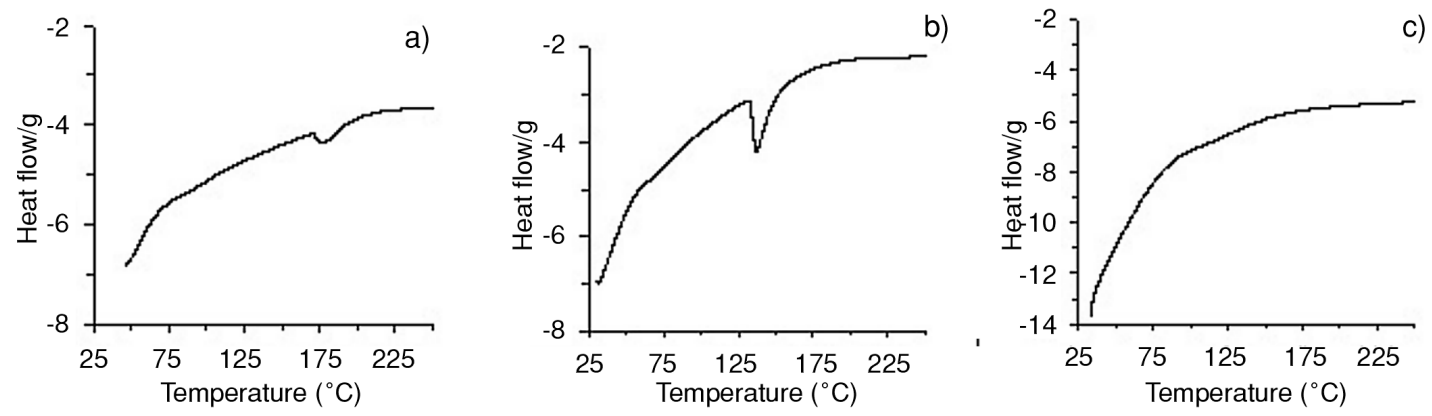

Figure 2. DSC curves of blank PVA filament (a), Loratadine (b) and loratadine loaded PVA filament (c).

Tables 2. Values of mechanical properties, drug content and enhancement factor of different treated filaments.

\begin{tabular}{|c|c|c|c|c|c|}
\hline $\begin{array}{c}\text { Formulation } \\
\text { code }\end{array}$ & $\begin{array}{c}\text { Tensile } \\
\text { strength (MPa) }\end{array}$ & $\begin{array}{c}\text { Young's } \\
\text { modulus (MPa) }\end{array}$ & $\begin{array}{c}\text { Stiffness } \\
(\mathrm{KN} / \mathrm{m})\end{array}$ & $\begin{array}{c}\text { Drug loading } \\
(\mathrm{mg} / 0.5 \mathrm{~g} \text { of filament) }\end{array}$ & $\begin{array}{c}\text { Enhancement } \\
\text { factor (times) }\end{array}$ \\
\hline $\begin{array}{c}\text { F0 (unloaded } \\
\text { filament) }\end{array}$ & $20.13 \pm 0.38$ & $1931.5 \pm 193.1$ & $134.8 \pm 11.8$ & & \\
\hline F1 & $19.81 \pm 0.25$ & $1727.8 \pm 434.3$ & $111.7 \pm 26.6$ & $0.19 \pm 0.03$ & - \\
\hline F2 & $19.3 \pm 0.68$ & $1630.2 \pm 137.2$ & $99.8 \pm 8.3$ & $2.33 \pm 0.14$ & 12.2 \\
\hline F3 & $20.5 \pm 0.53$ & $1633.4 \pm 307.9$ & $100.04 \pm 18.8$ & $1.33 \pm 0.10$ & 7.0 \\
\hline F4 & $19.95 \pm 0.34$ & $1468.9 \pm 109.8$ & $89.97 \pm 6.72$ & $4.49 \pm 0.19$ & 23.6 \\
\hline F5 & $20.16 \pm 0.26$ & $1475.3 \pm 279.6$ & $86.48 \pm 5.2$ & $4.00 \pm 0.21$ & 21.1 \\
\hline F6 & $19.08 \pm 0.33$ & $1531.5 \pm 340.5$ & $93.8 \pm 4.95$ & $1.43 \pm 0.13$ & 7.5 \\
\hline F7 & $20.82 \pm 0.24$ & $1653.5 \pm 26.5$ & $101.3 \pm 3.8$ & $1.56 \pm 0.13$ & 8.2 \\
\hline F8 & $20.61 \pm 0.33$ & $1712.7 \pm 63.2$ & $104.9 \pm 3.8$ & $1.40 \pm 0.09$ & 7.3 \\
\hline
\end{tabular}




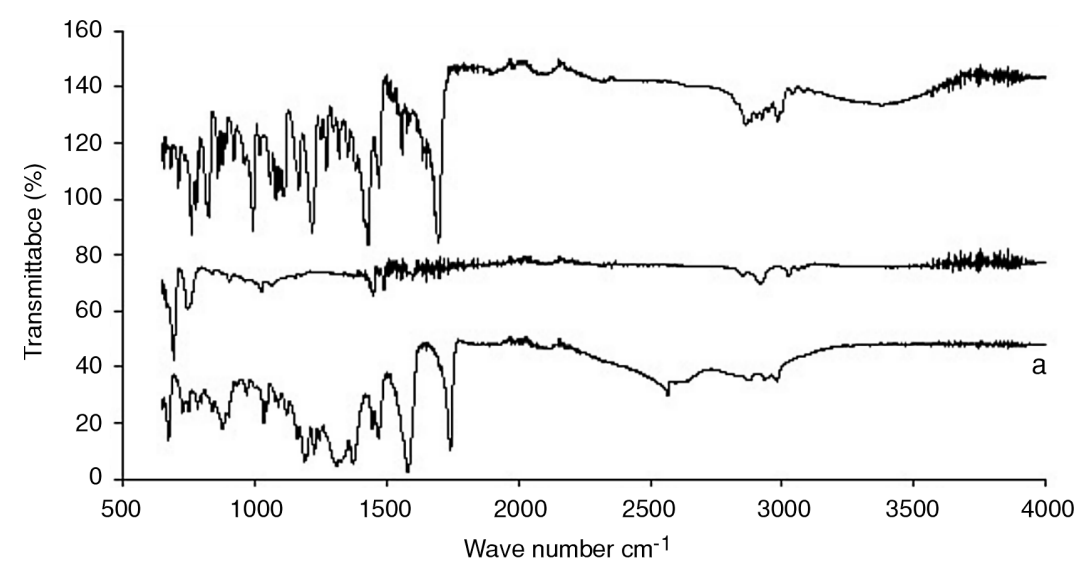

Figure 3. FTIR spectra of Loratadine (a) blank PVA filament (b) and loratadine loaded PVA filament (c).

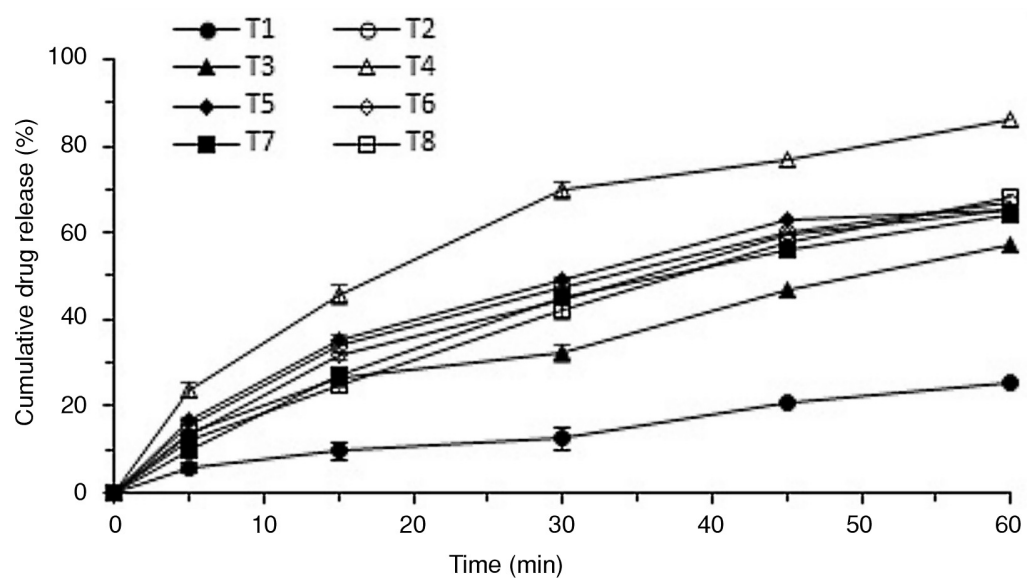

Figure 4. In-vitro release of loratadine from different printed tablets.

larly of soluplus and glycerin. The microwave treated filament (F8) has shown almost similar changes in mechanical properties as those treated with solubilizer.

\section{Drug content in the loaded filaments}

The drug content in PVA filament $(0.5 \mathrm{~g}$ which was almost equivalent to one printed tablet of given dimensions) was measured using UV-spectrophotometer and has been summarized in Table 2. The formulation $\mathrm{F} 1$ contains $0.19 \pm 0.03 \mathrm{mg}$ loratadine which indicates $\sim 0.04 \%$ of loading. These results are in agreement with previous studies showing the drug loading of $<1 \%(6-8)$. The formulation con- taining solubilizer (Soluplus or SLS) F2 and F3 have demonstrated 2.33 and $1.33 \mathrm{mg}$ loratadine, respectively. These results showed a 7 and 12 times enhancement of drug loading in the filament following the inclusion of soluplus and SLS, respectively. The drug content measured for F4-F5 was 4.0 and $4.49 \mathrm{mg}$, respectively that counts $>20$ times higher drug loading as compared with the control i.e. F1. This indicated that the pretreatment with glycerin results in a significant increase in drug loading i.e. twice in case of F4 while three times in case of F5 (as compared to their counterparts F2 and F3). While the formulations pretreated with PEG-400 i.e. F6 and F7 have shown the drug content of 1.43 and 
1.56 which was 7 to 8 times higher than the control. This indicated that the pretreatment with PEG-400 did not result in any enhancement in drug loading as compared with the counterparts F2 and F3, respectively. The filament microwaved before soaking in drug solution (F8) has also shown $\sim 7$ times enhancement in drug loading as compared with untreated filament (F1). Hence, the formulation pretreated with glycerin followed by soaking in drug solution containing soluplus (i.e. F4) was optimal in terms of drug loading.

\section{Differential Scanning Calorimetry}

DSC curve of blank filament has shown a step like endothermic event in the temperature range of $175-190^{\circ} \mathrm{C}$ (Fig. 2a). Loratadine (Fig. 2b) has shown an endothermic peak near $134^{\circ} \mathrm{C}$ corresponding to the melting of the drug. The drug-loaded filament (Fig. 2c) however has not shown any endothermic event. This might be due to the fact that the amount of drug in the loaded filament is too small to be detected by the sensitive range of the instrument. However, the absence of any degradation signal in Figure $2 b$ after melting of drug suggests that it is thermally stable at temperature till $250^{\circ} \mathrm{C}$ hence useful for 3D printing through FDM which requires temperature usually around $220^{\circ} \mathrm{C}$.

\section{ATR-FTIR}

The IR spectrum of the loratadine showed two characteristic bands between 1550 to $1710 \mathrm{~cm}^{-1}$ (as shown in Fig. 3a) which are attributed to amide and ester group (site with a high probability of intermolecular interaction) within the drug molecule (Frizon et al. 2013). On the other hand, no such peaks were seen in this range in plain PVA filaments (Fig. 3b). Furthermore, the drug-loaded filaments showed the peaks characteristics of the drug which suggested that the incorporation of a drug in the PVA filament did not cause any chemical shift (as shown in Fig. $3 \mathrm{c})$.

\section{Characteristics of 3D printed tablets}

The drug-loaded filaments (F1-F8) were successfully printed into tablets (T1-T8) of given design features with weight recovery $>95 \%$ (Table 3 ). This confirms that these drug-loaded filaments remained suitable for insertion into $3 \mathrm{D}$ printer. The tablets have shown much higher hardness ( $>10$ $\mathrm{Kg} / \mathrm{cm}^{2}$ which is higher than the upper limit of hardness tester (15) and no sign of any friability (i.e. $0 \%$ ). This indicated a higher degree of strength of printed tablets to any mechanical breaking or fracture.

\section{Assay of 3D printed tablets}

Drug content per tablet as determined by UV assay (see Table 3) has a similar trend as seen in the case of respective filaments. The drug content of tablet formulation in decreasing order was T4 $>$ T5 $>\mathrm{T} 2>\mathrm{T} 6>\mathrm{T} 3>\mathrm{T} 8>\mathrm{T} 7>\mathrm{T} 1$. Herein the formulation prepared from filaments pretreated with glycerin and soaked in drug solution containing soluplus (i.e. T4) provided the highest drug content per tablet.

\section{Drug release from 3D printed tablets}

Dissolution results of 3D printed tablets of T1 formulation have shown $\sim 10 \%$ drug release in the first $30 \mathrm{~min}$. While tablets of T2-T8 exhibited $30 \%$ to $70 \%$, release in $30 \mathrm{~min}$. (Fig. 4). The drug release of all formulations was according to following order $\mathrm{T} 4>\mathrm{T} 5>\mathrm{T} 2>\mathrm{T} 7>\mathrm{T} 6>\mathrm{T} 8>\mathrm{T} 3>\mathrm{T} 1$.

According to the release modeling, $\mathrm{T} 1$ followed the first-order release while all other tablet formulations followed the Higuchi model of drug release with the value of ' $n$ ' $>0.5$ (indicative of non-

Table 3. Estimated and actual weight, accuracy in weight and drug content per tablet of various formulations of printed tablets.

\begin{tabular}{|c|c|c|c|c|}
\hline $\begin{array}{c}\text { Formulation } \\
\text { code }\end{array}$ & $\begin{array}{c}\text { Estimated } \\
\text { weight }(\mathrm{mg})\end{array}$ & $\begin{array}{c}\text { Actual weight } \\
(\mathrm{mg})\end{array}$ & $\begin{array}{c}\text { Weight } \\
\text { accuracy }(\%)\end{array}$ & $\begin{array}{c}\text { Drug content } \\
\text { of each tablet }(\mathrm{mg})\end{array}$ \\
\hline T1 & 480 & $463.3 \pm 5.7$ & 96.5 & 0.11 \\
\hline $\mathrm{T}$ & 480 & $475.5 \pm 4.2$ & 99.06 & 11 \\
\hline T3 & 480 & $473.2 \pm 1.8$ & 98.3 & 3.97 \\
\hline T4 & 480 & $474.6 \pm 4.7$ & 98.9 & 3.85 \\
\hline T5 & 480 & $479.8 \pm 1.1$ & 99.9 & 1.23 \\
\hline T6 & 480 & $474.5 \pm 2.6$ & 98.8 & 1.16 \\
\hline T7 & 480 & $470.2 \pm 4.6$ & 97.9 & 101.1 \\
\hline T8 & 480 & $485.6 \pm 2.1$ & & 1.20 \\
\hline
\end{tabular}


Fickian release) except T4 which exhibited $\mathrm{n}<0.5$ hence showed the Fickian release.

\section{DISCUSSION}

FDM based three-dimensional printing utilizes pre-fabricated filaments or tailor-made filament for the preparation of customized medicine. The former having excellent printability have largely been replaced by the tailor-made filaments due to poor drug-loading efficiency (13). The diameter, surface morphology and stiffness of pre-fabricated filaments are the key factors that determine their printability through FDM based 3D printers. In the present study, careful manipulation of these properties was done by the addition of solubilizing aid (plasticizer and solubilizer) for the enhancement of drug-loading efficiency of PVA filament without compromising the printability.

The changes in surface texture on treatment with drug loading solution have permitted more drug adsorption on the rough filaments (16) and contributed to higher drug loading of these filaments. The reduction in values of elastic modulus $(\sim 25 \%)$ and stiffness $(\sim 35 \%)$ as observed in those filaments containing solubilizer and/or plasticizer (or treatment with microwave energy) indicated the plasticizer effect of these substances or treatment resulting in the increase of overall solubility of drug and changes the molecular packing of filament making it more diffusible for the drug (17). This subsequently has caused an increased penetration of drug solution into the filament core, hence the enhanced drug loading. The highest change ( $\sim 24$ times higher drug content) was observed in the case of formulation that was pretreated with glycerin before soaking in drug solution containing solubilizers (Soluplus or SLS). This certainly strengthens the argument that loosening of molecular packing by plasticizers followed by solubilizing action of solubilizers (18) resulted in the enhancement of the drug-loading efficiency of PVA filaments.

FTIR results have shown the absence of any significant chemical interaction while DSC analysis indicated the thermal stability of drugs during the drug loading and printing process, therefore the loaded drug content remains freely available for release from the printed tablets.

The drug contents of tablets of all formulations were almost similar to precursor filaments, indicating the efficiency of 3D printing process in preserving the contents completely for a thermostable drug as in the case of loratadine used in this study (12). The drug release from the printed tablets followed diffusive or erosive mechanisms. This indicated that the changes in the inner molecular packing and surface roughness that occur during drug loading has allowed easy elution.

\section{CONCLUSION}

The study concludes that the treatment with solubilizing aids (glycerin as plasticizer and Soluplus as solubilizer has significantly improved the drug loading efficiency of pre-fabricated PVA filament without compromising their printability.

\section{Acknowledgments}

Authors would like to thank the Higher Education Commission (HEC), Pakistan for funding this project under National Research Projects for Universities (NRPU) scheme 7610/Punjab/NRPU/ R\&D/HEC/2017.

\section{Conflict of interest}

The authors declare no conflicts of interest.

\section{REFERENCES}

1. Acosta-Vélez G.F., Wu B.M.: Polym. Sci. 2, 10 pages (2016).

2. Goldberger J.J., Buxton A.E.: JAMA 309, 2559 (2013).

3. Ventola C.L.: Pharm. Ther. 39, 704 (2014).

4. Stansbury J.W., Idacavage M.J.: Dent. Mater. 32, 54 (2016)

5. Sadia M., Sośnicka A., Arafat B., Isreb A., Ahmed W., et al.: Int. J. Pharm. 513, 659 (2016).

6. Goyanes A., Buanz A.B.M., Basit A.W., Gaisford S.: Int. J. Pharm. 476, 88 (2014).

7. Skowyra J., Pietrzak K., Alhnan M.A.: Euro. J. Pharm. Sci. 68, 11 (2015).

8. Goyanes A., Buanz A.B.M., Hatton G.B., Gaisford S., Basit A.W.: Euro. J. Pharm. Biopharm. 89, 157 (2015a).

9. Goyanes A., Robles Martinez P., Buanz A.B.M., Basit A.W., Gaisford S.: Int. J. Pharm. 494, 657 (2015b).

10. Goyanes A., Chang H., Sedough D., Hatton G.B., Wang J., et al.: Int. J. Pharm. 496, 414 (2015c).

11. Okwuosa T.C., Stefaniak D., Arafat B., Isreb A., Wan K.W., Alhnan M.A.: Pharm. Res. 33, 2704 (2016).

12. Pietrzak K., Isreb A., Alhnan M.A.: Euro. J. Pharm. Biopharm. 96, 380 (2015). 
13. Solanki N.G., Tahsin M., Shah A.V., Serajuddin A.T.M.: J. Pharm. Sci. 107, 390 (2018).

14. Melocchi A., Parietti F., Maroni A., Foppoli A., Gazzaniga A., Zema L.: J. Drug Deliv. Sci. Technol. 30, 360 (2015).

15. Zhang J., Feng X., Patil H., Tiwari R.V., Repka M.A.: Int. J. Pharm. 519, 186 (2017).
16. Alhijjaj M.,Belton P., Qi S.: Euro. J. Pharm. Biopharm. 108, 111(2016).

17. Mishra A., Pathak A.K.: Curr. Res. Pharm. Sci. 7, 1 (2017).

18. Lim H., Hoag S.W.: AAPS PharmSciTech 14, 903 (2013).

(C) 2020 by Polish Pharmaceutical Society. This is an open access article under the CC BY NC license (http://creativecommons.org/licenses/BY/4.0/). 